\title{
Single longitudinal mode fiber ring laser
}

\author{
Luis Rodriguez-Cobo, Ruben Ruiz-Lombera, M. Angeles Quintela, \\ Jesus Mirapeix and Jose-Miguel Lopez-Higuera \\ Photonics Engineering Group, Universidad de Cantabria, Santander, 39005, Spain
}

\begin{abstract}
In this work, a dual-wavelength fiber ring laser working on the SLM regime is proposed and experimentally verified. A proof-of-concept device has been tested employing high precision small-sized SLM fiber lasers to complement the feedback produced by a passive FBG. The ring laser output inherits the spectral benefits of its SLM seed lasers but maintaining some power-related properties of ring cavities. These spectral benefits can also be achieved for a single wavelength, simplifying the requirements regarding power equalization between different wavelengths to obtain the SLM regime. The experimental results exhibit a remarkably good wavelength stability and SLM operation of a fiber ring laser with several meters of cavity length, both with single and multi-wavelength configurations.
\end{abstract}

Keywords: Fiber Lasers; Erbium; Laser stability; Fiber Bragg Gratings

\section{Introduction}

Several works on multi-wavelength fiber lasers have been published in the last years, partly because of their great potential for some applications like microwave photonics systems, fiber-optic sensors, spectroscopy and wavelength

5 division multiplexed systems [1, 2, 3]. A configuration of erbium-doped fiber ring laser is an interesting choice to generate a multiple wavelength output due to its good compatibility with other optical fiber components, high gain and low noise figure.

Preprint submitted to Journal of Optics and Laser Technology

September 6, 2017

(C) 2018. This manuscript version is made available under the CC-BY-NC-ND 4.0 license http://

creativecommons.org/licenses/by-nc-nd/4.0/ 
However, mainly due to the long lasing cavity and unstable mode compewith different lasing wavelengths are used as seeds in a typical fiber ring laser structure with a fiber Bragg grating (FBG) as filter. A very narrow filtering technique based on the spectral overlap of uniform FBGs [10] has been employed to build the seed SLM linear fiber lasers. The proposed structure maintains the 35 SLM operation even with a single seed laser and is capable of delivering higher output power than isolated seeds. With a proper sizing, this configuration can be very useful to translate power variations produced within the ring cavity to different stable lasing wavelengths simultaneously. 


\section{Laser configuration} active medium trough passive methods, typically allowing light to travel only in one direction. The cavity length of these lasers is usually long and their spectral properties are mainly driven by the feedback method (e.g. FBGs) thus, achieving SLM operation can be sometimes challenging. However, Erwavelenghts are active in a laser cavity. Based on these ideas, a DBR seed (or several) can be employed to force the ring cavity to lase with the same properties as the injected seed. The proposed configuration is depicted in Fig. 1 (bottom).

The fiber ring laser employs Er-doped fiber as active medium and the feedback is produced by a highly saturated uniform FBG centered to favor lasing in the Bragg wavelength and avoid peak gain resonances. A circulator is also employed to determine the amplification direction of the cavity. This structure produces a multimode laser whose spectrum is determined by the chosen FBG and narrowed by the gain competition of the Erbium-doped fiber.

55 using a linear stage based on Er-doped fiber to increase its peak power (Fig. 1, top), before launching it into the ring cavity. Since the initial power of the seed laser is higher than the resonance of the ring cavity, the whole cavity inherits the spectral properties of the seed laser, maintaining the SLM regime, but increasing the output power. Although this ring-based structure can be useful for specific applications, it becomes more interesting when different seed lasers are launched into the ring cavity, sharing the latter active medium. This setup is depicted in Fig. 1 (bottom), where two similar DBR seed lasers working at different wavelengths are employed. This way, more complex structures can 65 properties are individually maintained, even with different powers for each seed laser. 


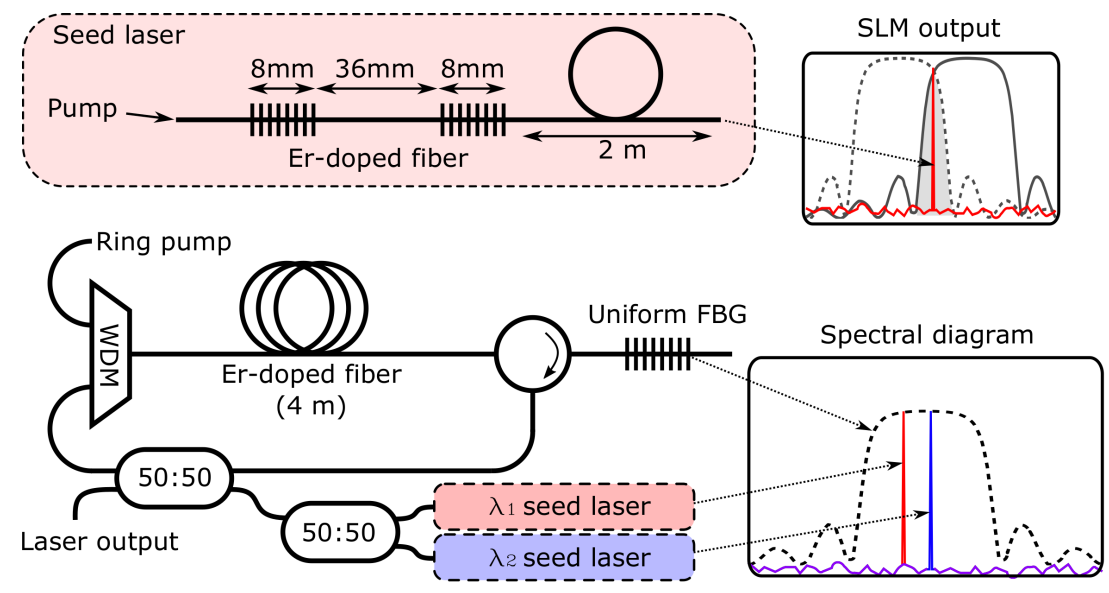

Figure 1: A SLM laser is amplified through $2 \mathrm{~m}$ of Er-doped fiber, obtaining a seed laser (top). Proposed ring configuration (bottom): different seed lasers are launched into the same active medium to obtain a common output.

\section{Experimental characterization of the seed laser}

Different seed lasers have been manufactured according to the structure depicted in Fig. 1, (top). For each laser, two FBGs of $L=8 \mathrm{~mm}$ have been inscribed into commercial Er-doped fiber (I-25 from Fibercore) using the phase mask technique with a continuum UV laser emitting at $244 \mathrm{~nm}$. One of the FBGs was partially post-exposed to adjust its central wavelength and reach the SLM regime [10]. After finishing the DBR structure, 2 meters of Er-doped fiber (I-25) were spliced after the small-sized DBR structure to increase its peak power (Fig. 1, top). One of these manufactured seed lasers has been experimentally characterized before launching it into the ring cavity.

In Fig. 2, several experimental properties of a SLM seed laser are depicted. The SLM operation (a) has been measured with a high resolution optical spectrum analyzer (BOSA-C from Aragon Photonics with a spectral resolution of $0.08 \mathrm{pm}$ ) and also employing a heterodyne detection system. In Fig. 2 (b), a single peak is shown, proving the SLM operation.

The heterodyne setup has been changed to the delayed self-heterodyne detection scheme [11], in order to measure the Full Width at Half Maximum 
(FWHM) linewidth of the emitted wavelength. A phase modulator (Photline MPZ-LN20) has been employed to perform the $5 \mathrm{GHz}$ modulation. A $150 \mathrm{~km}$ length standard optical fiber has been employed as the delayed line. An EDFA has been introduced in the setup to amplify the signal of the delayed line. According to [11], the real linewidth is the square root of two times the measured 90 FWHM, so the achieved linewidth was around $1 \mathrm{kHz}$ as shown in Fig. 2 (c). This narrow linewidth is comparable to previous results achieved with similar Er-doped fibers [10], suggesting a very stable SLM operation of the fiber laser.
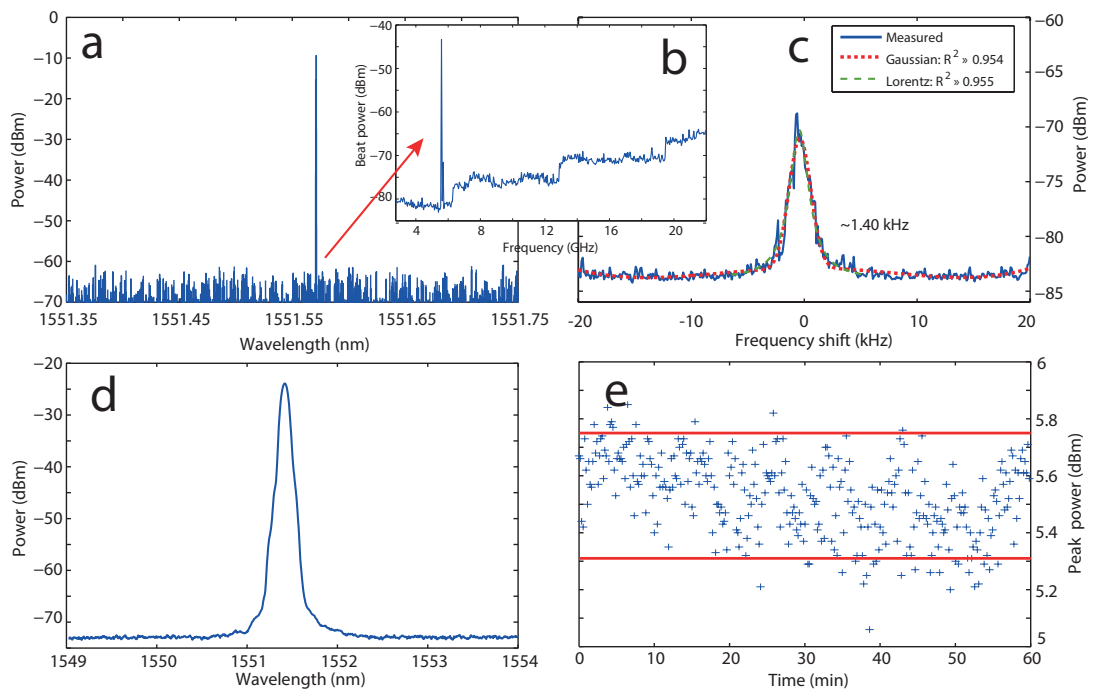

Figure 2: Experimental characterization of a SLM seed laser. SLM regime measured with BOSA-C (a) and heterodyne setup (b). Linewidth around $1 \mathrm{kHz}$ measured with delayed selfheterodyne detection scheme (c). OSNR around 48dB measured with OSA (d). Power stability under $0.44 \mathrm{~dB}$ measured for $60 \mathrm{~min}$ (e) while pump power was maintained at $100 \mathrm{~mW}$.

The output laser signal has been directly connected to an OSA (HP70952B, that exhibits a wavelength resolution of $60 \mathrm{pm}$ ) to study the power behavior of the manufactured laser. Maintaining the pump current constant, the generated output optical power has been monitored during 60 min using the OSA. The power stability measured each 30 seconds (Fig. 2, e) is depicted, achieving a drift of $0.44 \mathrm{~dB}$ with a $90 \%$ confidence interval. For the pump current employed during the stability test, the output laser exhibited an optical signal-to-noise 
ratio (OSNR) higher than $48 \mathrm{~dB}$ (Fig. 2, d).

\section{Experimental characterization of the ring laser}

After the characterization of an isolated SLM seed laser, the ring configuration depicted in Fig. 1 (bottom) has been mounted employing 4 meters of Er-doped fiber (I-25 from Fibercore). Two seed lasers have also been connected to the cavity while the chosen FBG $(L=24 \mathrm{~mm})$ centered at these wavelengths $\left(\lambda_{c} \approx 1551.5 \mathrm{~nm}\right)$ that exhibits a high bandwidth $\left(B W_{3 d B} \geq 2 \mathrm{~nm}\right)$.
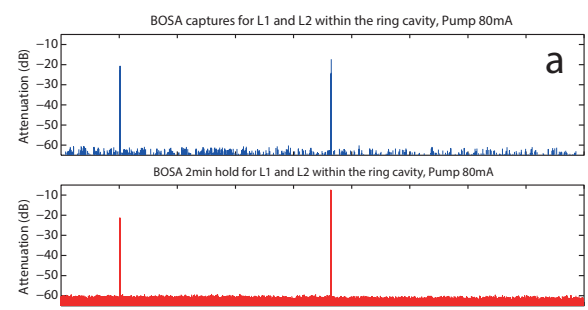

BOSA capture for only L1 within the ring cavity, Pump $80 \mathrm{~mA}$
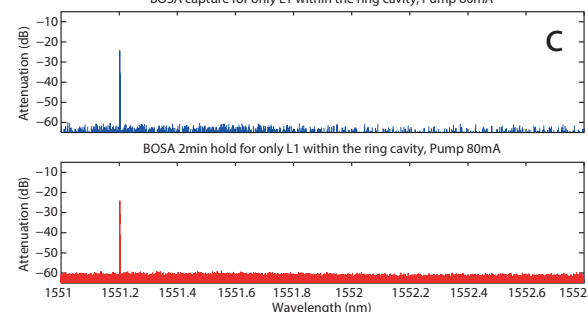

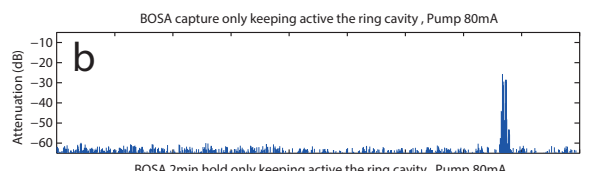

BOSA 2 min hold only keeping active the ring cavity, Pump $80 \mathrm{~mA}$

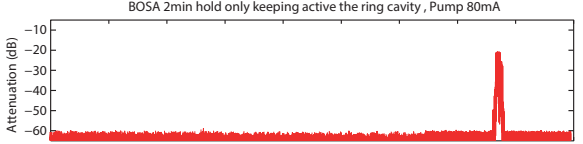

BOSA capture for only L2 within the ring cavity, Pump $80 \mathrm{~mA}$
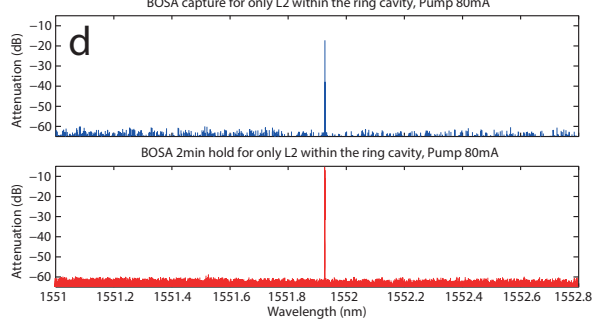

Figure 3: BOSA-C instantaneous captures (top) and 2 minute hold (bottom) of: two seed lasers (a); no seed laser (b); only $\lambda_{1}$ seed laser (c); only $\lambda_{2}$ seed laser (d). All scenarios have been also verified using the heterodyne method. The pump power of both seed lasers was set around $150 \mathrm{~mW}$.

\subsection{Wavelength measurements}

The wavelength evolution of the most common operation situations has been analyzed using the high resolution optical spectrum analyzer (BOSA-C) and verified using the heterodyne method. For each of the presented results, two similar graphs are depicted: the first one corresponds to an instantaneous measurement, while the second one shows a 2 minutes hold of the laser signal. In 
the first case depicted in Fig. 3 (a), two seed lasers have been injected into the ring cavity with the same power, while the ring pump was active. Both seed lasers maintain their SLM regime after being amplified by the ring cavity. The second scenario (b) exhibits the resonance associated with the feedback FBG that creates a multimode laser when no seed was injected. The multimode laser achieved without seed lasers exhibits a much narrower bandwidth than the passive response of the FBG mainly provoked by the Erbium gain competition. The remaining cases depicted in Fig. 3 are associated with single seed injection: only $\lambda_{1}$ seed (c) and only $\lambda_{2}$ seed (d). Those cases are the worst-case scenario in terms of the incoming power difference and both lasers maintain their original SLM regime.

Other remarkable parameter related to high precision lasers is the linewidth.

125 The delayed self-heterodyne detection scheme [11] has been employed again to verify the influence of the ring cavity on the FWHM of the laser outputs. Both seed lasers have been experimentally characterized before and after being injected into the ring cavity using the same setup (phase modulator, $150 \mathrm{Km}$ as delay and an EDFA to overcome the power reduction). Both achieved linewidths were around $1 \mathrm{kHz}$, proving that the ring output maintains the spectral properties of the seed laser.

\subsection{Power measurements}

Although wavelength stability mainly depends on seed lasers, their output power is more affected by the ring cavity because all wavelengths must share the active medium. The same power stability measurements have been repeated for two simultaneous seed lasers maintaining the ring pump current. The generated output optical power was monitored each 30 seconds during 60 min using the OSA. Each laser peak has been independently analyzed and the achieved results are depicted in Fig. 4 (a, $\lambda_{1}$ seed; b, $\lambda_{2}$ seed). As expected, their power stability is worse than the isolated seed lasers, however they still remain under $2 \mathrm{~dB}(90 \%)$ even without being isolated from environmental vibrations.

As commented in the previous section, the spectral properties of the seed 
lasers are maintained independently of their power ratio: the ring laser working on SLM regime can be achieved even with a single seed. In Fig. 4 (c), power measurements captured with an OSA are depicted for each extreme case: one seed laser at a time and no seed laser. Each seed laser has been injected with the same power, being amplified the same value for the ring cavity $\left(G_{\text {ring }} \approx 3 d B\right)$ while keeping their original spectral properties. However, there are some limitations regarding the ratio between the power pumped into the ring cavity and the power of the seed lasers. In Fig. 4 (d), power traces have been captured for different powers pumped into the ring cavity. As the ring power increases, the output lasers are more affected by the ring cavity, but they maintain their seed spectral properties. However, when the pump is excessive, in addition to amplifying the seed lasers, the ring cavity starts to lase at other wavelengths different from those of the seeds. Although the spectral properties of the seed lasers are still maintained, they can be superposed with other multimode lasers directly created within the cavity, adding extra noise to the system. This problem can be minimized with a proper ring cavity design taking into account the power injected by the seed lasers or employing another secondary FBG to create those multimode resonances away from the desired wavelengths.

\section{Conclusion}

In this work, a seed-aided fiber ring laser working on SLM regime has been proposed and experimentally characterized. This scheme combines a typical fiber ring laser structure with a FBG for filtering with high quality small-sized SLM seed lasers manufactured directly in commercial Er-doped fiber to improve the final spectral properties. The spectral properties of the output lasers are mainly driven by the seed lasers employed by forcing the feedback process with an active laser signal instead of passive FBGs. Although each laser wavelength inherits the spectral properties of the seed, since they share a last amplification stage within the same active medium, they also exhibit some ring-associated properties mainly related to their output power. The achieved results have 

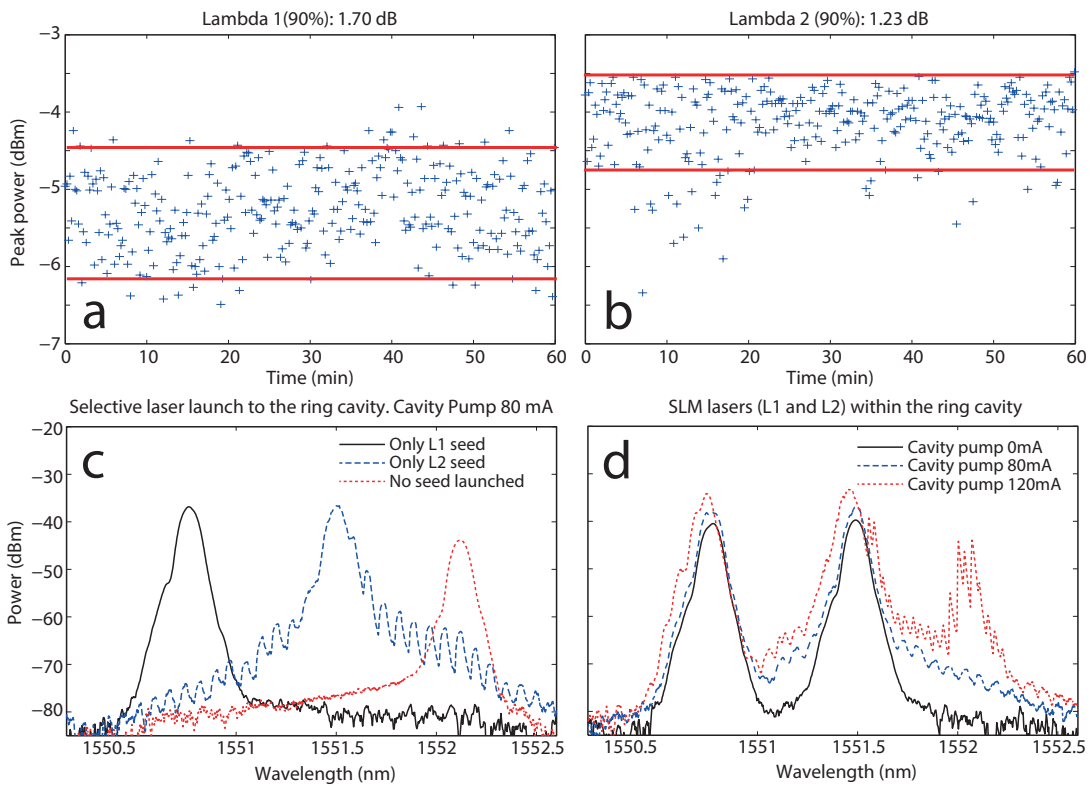

Figure 4: Ring cavity power measurements. Power stability measured at ring output of $\lambda_{1}$ (a) and $\lambda_{2}$ (b) seed lasers. The pump power of seed lasers is adjusted to launch the same power at both wavelengths (around $150 \mathrm{~mW}$ ). OSA traces for selective seed injection (c) and OSA traces for different ring pump power (d) while seed lasers are active.

proved that this technique is able to obtain dual-wavelength ring lasers working on the SLM regime with different power levels for each wavelength, reaching also the SLM regime for a single wavelength output. Although some limitations regarding the maximum cavity pump power have been found, they can be overcome by a proper cavity design or by forcing the ring self-resonances out of the wavelength of interest. Thus, with a proper sizing of cavities, a laser emitting at higher powers while maintaining the spectral properties of their seeds can be achieved.

\section{Acknowledgments}

This work has been supported by the project TEC2016-76021-C2-2-R of the Spanish Government cofunded by EU/FEDER funds. Special thanks to Fibercore for their support. 


\section{References}

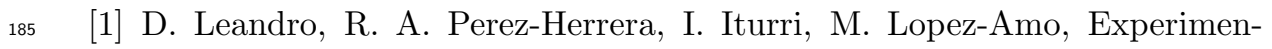
tal study of the slm behavior and remote sensing applications of a multiwavelength fiber laser topology based on dwdms, Applied Physics B 118 (3) (2015) 497-503.

[2] S. Rota-Rodrigo, I. Ibanez, M. Lopez-Amo, Multi-wavelength fiber laser in single-longitudinal mode operation using a photonic crystal fiber sagnac interferometer, Applied Physics B 110 (3) (2013) 303-308.

[3] U. Sharma, C.-S. Kim, J. U. Kang, N. M. Fried, Highly stable tunable dualwavelength q-switched fiber laser for dial applications, in: Laser Applications to Chemical and Environmental Analysis, Optical Society of America, p. MB3.

[4] X. Chen, J. Yao, Z. Deng, Ultranarrow dual-transmission-band fiber bragg grating filter and its application in a dual-wavelength single-longitudinalmode fiber ring laser, Optics letters 30 (16) (2005) 2068-2070.

[5] J. Liu, J. Yao, J. Yao, T. H. Yeap, Single longitudinal mode multiwavelength fiber ring lasers, in: Optical Fiber Communication Conference, Optical Society of America, p. ThB2.

[6] J. Sun, X. Yuan, X. Zhang, D. Huang, Single-longitudinal-mode dualwavelength fiber ring laser by incorporating variable saturable absorbers and feedback fiber loops, Optics communications 273 (1) (2007) 231-237.

[7] Z. Lu, C. Grover, A widely tunable narrow-linewidth triple-wavelength erbium-doped fiber ring laser, Photonics Technology Letters, IEEE 17 (1) (2005) 22-24.

[8] M. P. Fok, C. Shu, Tunable dual-wavelength erbium-doped fiber laser stabilized by four-wave mixing in a 35-cm highly nonlinear bismuth-oxide fiber, Optics express 15 (10) (2007) 5925-5930. 
[9] S. Pan, X. Zhao, C. Lou, Switchable single-longitudinal-mode dualwavelength erbium-doped fiber ring laser incorporating a semiconductor optical amplifier, Optics letters 33 (8) (2008) 764-766.

[10] L. Rodriguez-Cobo, M. Quintela, S. Rota-Rodrigo, M. Lopez-Amo, J. M. Lopez-Higuera, Single-longitudinal mode laser structure based on a very narrow filtering technique, Optics express 21 (8) (2013) 10289-10294.

[11] T. Okoshi, K. Kikuchi, A. Nakayama, Novel method for high resolution measurement of laser output spectrum, Electronics letters 16 (16) (1980) 630-631. 